\title{
THE COUPLING OF ELECTROMAGNETIC POWER TO PLASMAS
}

\author{
F. Louche \& R. Koch \\ Plasma Physics Laboratory \\ Ecole Royale Militaire - Koninklijke Militaire School \\ 1000 Brussels - BELGIUM; EURATOM-Belgian State Association
}

\begin{abstract}
This lecture addresses, on the basis of elementary and intuitive treatment, the process of coupling of electromagnetic power to plasma. Coupling is here meant in a broad sense. It consists of four different steps. (i) The first one is the coupling of vacuum electromagnetic power to plasma waves. An elementary antenna coupling theory is given. The state of the art in coupling models and status of comparisons with experiments are briefly discussed. (ii) The second is the transfer of plasma wave energy to particle energy. The resonant processes leading to this transfer are described in a heuristic way. (iii) The third one is the build-up of fast particle populations. It will be outlined through a sketch of quasilinear diffusion for the simple case of Landau damping. (iv) The last step is the conversion of power through the resonant particle population to bulk plasma heating by collisions, which will be briefly addressed.
\end{abstract}

\section{INTRODUCTION}

The principle of wave heating is similar for all schemes and is sketched in Fig.1. The electromagnetic energy is produced by a generator and sent to the machine area via transmission lines constituted of coaxial lines at low frequency and waveguides at higher frequency. At very high frequency optical transmission is also possible. Some matching circuitry has to be incorporated in the transmission system in order to prevent the reflected power to come back to the generator. The transmission line is connected to some launching structure (antenna, waveguide,) that will couple the power inside the machines vacuum chamber. The vacuum wave that exists inside the launching structure and at the very edge of the plasma is then converted to a plasma wave that transports electromagnetic energy to some region inside the plasma where it will be absorbed. This is the region where the resonant process occurs. This process accelerates the population of particles that is in resonance with the wave, usually a small fraction of the plasma particles. A slightly or strongly non-maxwellian resonant population builds up against the restoring force of collisions between this population and the remainder of the plasma. It is through the latter collisional process that the bulk of the plasma is heated up. In this paper, we shall not describe the technical parts of the launching systems, i.e. generators, transmission lines, matching systems (see lecture by Dumortier [2]), but shall focus on the physics of power coupling. Let us first start with the elementary wave theory.

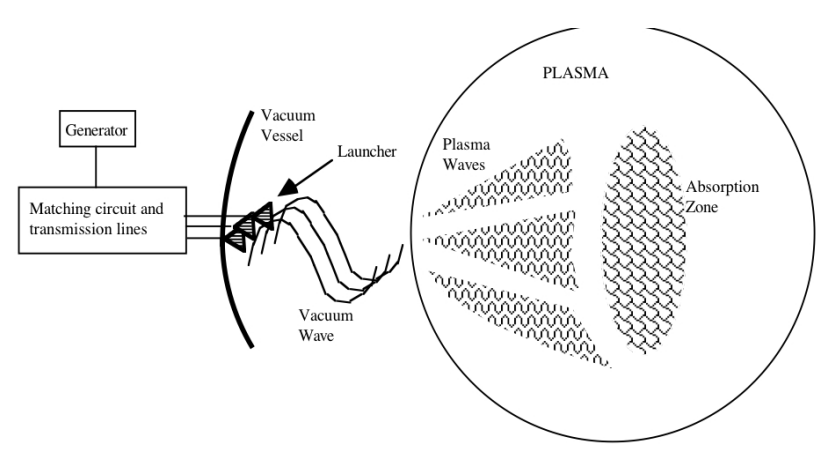

Figure 1: Principle of heating by plasma waves

\section{WAVE PROPAGATION IN A PLASMA}

The study of plasma waves is a very broad subject and we will limit ourselves to to the cold-plasma approximation [1] which is mostly sufficient to describe the basic propagation properties of the waves used in plasma heating. Interested readers should consult references $[8,9]$ for an exhaustive description of plasma waves.

We consider a plasma immersed in a static magnetic induction field $\overrightarrow{B_{0}}$. If the wave is considered as a first-order perturbation with a harmonic spacetime dependence $\sim \exp [-i(\omega t-\vec{k} \cdot \vec{x})]$ then the coldplasma dispersion relation can be written[8]:

$$
\mathbf{D} \cdot \vec{E}=\vec{k} \times(\vec{k} \times \vec{E})+\frac{\omega^{2}}{c^{2}} \varepsilon \cdot \vec{E}=0
$$

where $\vec{k}$ is the wave vector, $\vec{E}$ is the wave electric field, $\omega$ is the generator angular frequency and $c$ is the speed of light. It is sometimes useful to work instead with the refractive index vector $\vec{n}=\vec{k} / k_{0}$ where $k_{0}=\omega / c$ 
is the vacuum wave vector. The components of the cold-plasma dielectric tensor $\varepsilon$ are given by [8]:

$$
\varepsilon=\left(\begin{array}{ccc}
\varepsilon_{\perp} & i \varepsilon_{x y} & 0 \\
-i \varepsilon_{x y} & \varepsilon_{\perp} & 0 \\
0 & 0 & \varepsilon_{\|}
\end{array}\right) \text {with } \begin{aligned}
& \varepsilon_{\perp}=1-\sum_{s y} \frac{\omega_{p s}^{2}}{\omega^{2}-\omega_{c s}^{2}} \\
& \\
& \varepsilon_{\|}=1-\sum_{s} \frac{\omega_{p s}^{2} \Omega_{c s}}{\left(\Omega_{c s}^{2}-\omega^{2}\right) \omega} \\
& \frac{\omega_{p s}^{2}}{\omega^{2}}
\end{aligned}
$$

The wave equation is an algebraic homogeneous system of 3 equations for the 3 cartesian components of the electric field. It will have a non vanishing solution if and only if the determinant of $\mathbf{D}$ is zero. This defines the dispersion relation, an implicit relation between the generator frequency, the parallel and perpendicular components of the refractive index vector $\overrightarrow{n_{\perp}}$ and $n_{\|}$, and the plasma properties (encoded in the dielectric tensor). The dispersion equation solution determines all the wave modes which can propagate in the plasma for a given plasma model. If the static magnetic induction $\overrightarrow{B_{0}}$ is taken along the $z$-direction of a cartesian frame, then, because of the isotropy in the plane parallel to $\overrightarrow{B_{0}}$, the direction of the perpendicular component of the wave vector $\overrightarrow{k_{\perp}}$ is irrelevant and can be arbitrarily chosen as $x$-direction. The dispersion equation is then expressed as a bi-quadratic equation in $n_{\perp}=\left|n_{\perp}\right|$ :

$$
\left|\begin{array}{ccc}
\varepsilon_{\perp}-n_{\|}^{2} & i \varepsilon_{x y} & n_{\perp} n \| \\
-i \varepsilon_{x y} & \varepsilon_{\perp}-n_{\perp}{ }^{2}-n_{\|}^{2} & 0 \\
n_{\perp} n \| & 0 & \varepsilon_{\|}-n_{\perp}^{2}
\end{array}\right|=0
$$

We shall generally express its solution as ${k_{\perp}}^{2}=$ $\kappa\left(k_{\|}, \omega\right)$ because $k_{\|}$is generally fixed by the launcher structure. The two roots are often called fast (or magnetosonic) and slow waves. Their behaviour will be studied in details in $[3,4]$ for the various range of frequencies. For our purpose we simply need to underline that at some frequencies (corresponding to specific locations in the plasma) "something can happen" ! First, the dielectric tensor becomes singular each time $\omega=\omega_{c s}$. This means that the particle current becomes infinite for a finite electric field (wave-particle resonances discussed in sections IVC,D, E). The other special frequencies are those for which $k_{\perp}{ }^{2} \rightarrow \infty$ and appear when $\varepsilon_{\perp}=0$. We speak about wave resonances and they will be discussed in section IV-B. In addition, at low frequencies -ion cyclotron range of frequencies or below- $\varepsilon_{\|}$is much larger than the other elements such that the dispersion equation reduces to the top left $2 \mathrm{X} 2$ determinant (3). This approximation corresponds to the zero electron mass limit and leaves only one wave (the fast wave) in the dispersion. In the ICRH the slow wave is evanescent almost everywhere in the plasma bulk, and practically the zero electron mass limit is equivalent to neglecting the parallel component of the electric field. This can be forced by setting up a screen in front of the launcher.

\section{ELEMENTARY WAVE COUPLING THEORY}

\section{A. Types of launcher and coupling}

The simplest case is that of electron cyclotron waves in large machines. In this case, the wavelength of the vacuum wave $\lambda=2 \pi / k_{0}$ is very small as compared to the plasma cross-section. The wave is launched as a propagating wave pencil that will progressively convert to a plasma wave. Because of the smallness of the wavelength, the boundary conditions at the conducting wall of the machine, as well as on the launching structure, play no explicit role. The wave can be accurately described in the geometric optics limit and the only boundary conditions that matter are the initial launching angle and reflections at the wall, if any.

If the vacuum wavelength becomes comparable to the antenna structure, the scale length of variations of edge plasma parameters or the plasma radius, the launcher environment and the plasma will affect the coupling process and a full boundary-value problem has to be solved to describe it. Such is usually the case of Alfvén wave, ion cyclotron, or lower hybrid wave launchers in medium or large-size machines. But this may also be the case of electron cyclotron launchers in low-field, small machines.

We shall now introduce coupling theory, on the basis of the simplest model. More sophisticated theories rest on similar principles but include more of a realistic geometry.

\section{B. Coupling model}

We consider the simplest case of an antenna facing a large plasma such that the plasma looks nearly uniform in the toroidal $(z)$ and poloidal $(y)$ directions (Fig.2). In the radial direction $x$ the plasma is usually non-uniform, and this is taken into account in standard coupling models, but here, in order to simplify the algebra, we consider a step model. The density is zero for $x<0$ and constant density for $\mathrm{x}$ positive. The influence of a realistic density profile was studied in [30]: the cut-off position and the density gradient were shown to have a primary importance in the assessment of an antenna coupling capabilities, but these considerations are out of the scope of the present introduction. We assume that the absorption is good and hence, there is no reflected wave. This is the so-called single-pass approximation. In addition, we shall also assume that the system is invariant in the y-direction (which implies in particular that the antenna is infinite) and neglect propagation in the $y$ direction $\left(k_{y}=0\right)$. Next, we have to choose a model for the plasma waves. To be specific, we choose the case of coupling in the ion cyclotron frequency range (ICRF). The slow wave being evanescent in the plasma bulk, we shall consider only coupling to the fast magnetosonic wave $(\mathrm{FW})$. As the $\mathrm{FW}$ equations will be 
needed later on, we first derive them, starting from the cold wave theory.

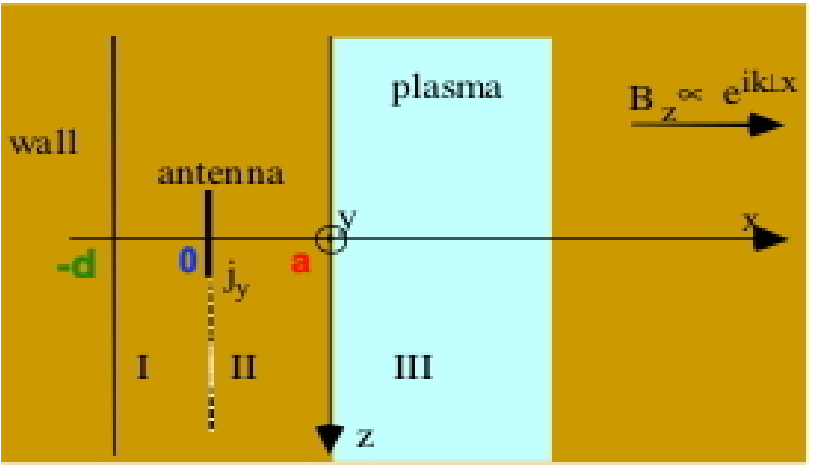

Figure 2: 2-D slab coupling model. The plasma is uniform in the $y$ (poloidal) and $z$ (toroidal) directions. The width of the antenna is $2 w_{z}$, the distance between the antenna and the plasma is $a$ and the distance between the antenna and the wall is $d$.

\section{The fast magnetosonic wave equation}

We write the full cold-plasma dispersion relation in terms of the parallel $\left(n_{\|}=n \sin \theta\right)$ and perpendicular $\left(n_{\perp}=N \cos \theta\right)$ components of the refractive index

$$
n_{\|}=\frac{k_{\|}}{k_{0}} ; n_{\perp}=\frac{-i}{k_{0}} \frac{d}{d x}
$$

We keep to $n_{\perp}$ its operator meaning because $x$ is the direction of inhomogeneity. In the parallel direction, the plasma is homogeneous and we use the Fourier transformed form. The dispersion equation (3) derived previously can be used and we take the zero electron mass limit to uncoupling the FW. This limit implies that the parallel electric field cannot penetrate the plasma, i.e. that the evanescence length of the slow wave is zero. Eq. (1) can be recast to

$$
\begin{aligned}
k_{\perp, F W}{ }^{2} E_{y} & =-i\left(\frac{d}{d x}+\mu k_{y}\right)\left(\omega B_{z}\right) \\
k_{\perp, F W}{ }^{2} E_{x} & =-\left(k_{y}+\mu \frac{d}{d x}\right)\left(\omega B_{z}\right) \\
i \omega B_{z} & =\frac{d}{d x} E_{y}-i k_{y} E_{x}
\end{aligned}
$$

with $\mu=\epsilon_{x y} k_{0}{ }^{2} / u, u=k_{0}{ }^{2} \varepsilon_{\perp}-k_{\|}^{2}$ and $k_{\perp, F W}{ }^{2}=$ $u\left(1-\mu^{2}\right)$. The other components of the FW field follow from Maxwell's equation $i \omega \vec{B}=\vec{\nabla} \times \vec{E}$ (with $\left.E_{z}=0\right)$ :

$$
B_{x}=\frac{-k_{\|}}{\omega} E_{y} ; \quad B_{y}=\frac{k_{\|}}{\omega} E_{x}
$$

Finally, taking the limit $k_{y} \rightarrow 0$ and eliminating $B_{z}$ from (5a)-(5c), we obtain the FW equation:

$$
\frac{d^{2} E_{y}}{d x^{2}}+k_{\perp, F W}{ }^{2} E_{y}=0
$$

\section{The Plasma surface impedance}

For a uniform plasma, the wave equation (7) has constant coefficient and the solutions are simply exponential. The single-pass approximation allows us to impose at $z \rightarrow \infty$ a radiating boundary condition and the wave solution in the plasma can be written:

$$
E_{y}=C_{I I I} \exp \left(i k_{\perp, F W} x\right)
$$

where $C_{I I I}$ is a constant (relative to region III in Fig. 2) to be determined. Eq. (6) then gives the $H_{z}$ field component:

$$
B_{z}=\frac{k_{\perp, F W}}{\omega} E_{y}
$$

The field in the plasma is thus known up to a multiplicative constant. Tangential field components being continuous at the plasma-vacuum interface (II-III), their ratio is also continuous. This quantity is known as the surface impedance of the plasma $Z_{S}$

$$
Z_{S}=\frac{E_{y}}{\omega B_{z}}=\frac{1}{k_{\perp, F W}}
$$

We express the continuity of this quantity at $x=0$ as

$$
\left[\left[Z_{S}\right]\right]_{0}=Z_{S}\left(0_{+}\right)-Z_{S}\left(0_{-}\right)=0
$$

In the general case where all field components are to be considered, the equivalent of (10) is a vector relation and $\mathbf{Z}_{S}$ is the surface impedance matrix:

$$
\left(\begin{array}{c}
E_{y} \\
E_{z}
\end{array}\right)=\mathbf{Z}_{S}\left(\begin{array}{c}
\omega B_{y} \\
\omega B_{z}
\end{array}\right)
$$

\section{E. Fields in the vacuum region I-II}

Equipped with this boundary condition, the vacuum problem can be solved on its own. The plasma properties will enter its solution only via the quantity $Z_{S}$ and the vacuum solution is therefore formally independent of the particular plasma model considered. The general electromagnetic field in vacuum can be decomposed into its TE (transverse electric) and TM (transverse magnetic) parts with respect to a given direction, here $z$. Maxwell's equations then appear in the form:

$$
\begin{aligned}
& \left(\begin{array}{c}
B_{x} \\
E_{y}
\end{array}\right)=\frac{1}{k_{0}{ }^{2}-k_{\|}^{2}}\left(\begin{array}{cc}
-i \omega / c^{2} & i k_{\|} \\
i k_{\|} & -i \omega
\end{array}\right)\left(\begin{array}{c}
i k_{y} E_{z} \\
d B_{z} / d x
\end{array}\right) \\
& \left(\begin{array}{c}
E_{x} \\
B_{y}
\end{array}\right)=\frac{1}{k_{0}^{2}-k_{\|}^{2}}\left(\begin{array}{cc}
-i \omega & i k_{\|} \\
-i k_{\|} & i \omega / c^{2}
\end{array}\right)\left(\begin{array}{c}
-i k_{y} B_{z} \\
d E_{z} / d x
\end{array}\right)
\end{aligned}
$$

$$
\frac{d^{2}}{d x^{2}}\left(\begin{array}{c}
E_{z} \\
B_{z}
\end{array}\right)=\left(k_{y}{ }^{2}+k_{\|}^{2}-{k_{0}}^{2}\right)\left(\begin{array}{c}
E_{z} \\
B_{z}
\end{array}\right)
$$

From this it can be seen that the problem can be solved independently for $E_{z}$ and $B_{z}$. The TM part 
of the field, which has a longitudinal (along $z$ ) $\vec{E}$ component does not couple to the plasma waves because $E_{z}=0$ in the plasma (see section II). Therefore, for the simplified problem considered here, we can retain the TE mode alone and ignore the field components deriving from $E_{z}$. The solution for the two vacuum regions is elementary:

$$
\begin{array}{ll}
\text { Region I: } & B_{z}=A_{I} \cosh k_{\|} x+B_{I} \sinh k_{\|} x \\
\text { Region II: } & B_{z}=A_{I I} \cosh k_{\|} x+B_{I I} \sinh k_{\|} x
\end{array}
$$

where the $A$ 's and the $B$ 's are constants to be determined by the following boundary conditions:

- At the metallic wall $x=-d: E_{y}=0$

- The antenna is represented by an infinitely thin current sheet of finite width $w$ and infinite length. This gives rise to a jump condition on the tangential magnetic field: $\left[\left[B_{z}\right]\right]_{0}=-\mu_{0} j_{y}$

- and a continuity condition $\left[\left[E_{y}\right]\right]_{0}=0$

- At the plasma surface, $x=0:\left[\left[Z_{s}\right]\right]_{a}=0$

These conditions are sufficient to determine the 4 constants in (15a) and (15b). In particular, this gives the relation between all field components and their source, the current density at the antenna $j_{y}[12]$.

There is one additional condition, the continuity of $E_{y}$ (or $B_{z}$ ) at $x=a$ that was not necessary to solve the vacuum problem. It can be used to determine $C_{I I I}$ in Eq. (8) as all field quantities in the vacuum region are now known.

\section{F. Poynting's theorem and antenna radiation}

Let us consider in the vacuum region I-II an arbitrary volume containing the antenna. Starting from Maxwells equations, one can easily write down Poynting's theorem [7] from which we get the complex radiated power in region II for an antenna current $\overrightarrow{j_{A}}$ :

$$
P=-\frac{1}{2} \int_{V} \vec{E} \cdot \overrightarrow{j_{A}} d V
$$

On the r.h.s. appears the work done by the electric field on the antenna current. Strictly speaking, it should be zero because the antenna is a metallic conductor on which the tangential electric field should vanish. It is non-zero because the current distribution on the antenna has been assumed rather than selfconsistently computed. This is known as the induced e.m.f. method. Though it may appear rough, this method usually gives good results if the assumed current is a reasonable guess of the exact one. In more sophisticated computations [16, 22], the current distribution on the antenna is self-consistently determined. A theory completely similar to the above one can be done for waveguide[16] or aperture launchers. In these cases, the incoming wave field distribution on the aperture is given and the field reflected by the plasma and surrounding structures is the result of the computation. Alternatively, the above formalism can be applied without changes if the aperture boundary condition is expressed as an equivalent current density[25]. It is to be observed that the quantity on the r.h.s. of Eq.(17) has both a real and an imaginary part. The real part is the power radiated by the antenna, while the imaginary part is related to the reactive properties of the antenna, as we will see. We can compute the former for our simplified model. First we rewrite Poyntings theorem (17) in terms of the $k_{\|}$field spectrum using Parseval's relation:

$$
P=-\frac{1}{2} \int_{V} E_{y} j_{y}{ }^{*} d V=-\frac{1}{4 \pi} \int_{-\infty}^{\infty} E_{y}\left(k_{\|}\right) j_{y}\left(k_{\|}\right) d k_{\|}
$$

The Fourier spectrum of the electric field $E_{y}\left(k_{\|}\right)$is evaluated in region II, in front of the strap $(x=0)$ and is given by:

$$
E_{y}\left(k_{\|}\right)=\omega \mu_{0}\left(g_{2}-i h_{2}\right) j_{y}\left(k_{\|}\right)
$$

with

$g_{2}-i h_{2}=\frac{i \sinh k_{\|} d}{k_{\|}} \frac{\sinh k_{\|} a-i Z_{S} \cosh k_{\|} a}{\sinh k_{\|}(a+d)-i Z_{S} \cosh k_{\|}(a+d)}$

The antenna radiation spectrum can be obtained from the Poynting theorem and taking the real part of it

$$
\Re(P)=-\frac{\omega \mu_{0}}{4 \pi} \int_{V} g_{2}\left|j_{y}\right|^{2} d k_{\|}
$$

If we take the limit $d \rightarrow \infty$

$$
\Re(P) \sim-\frac{\omega \mu_{0}}{4 \pi} \int_{V} \frac{k_{\perp}}{{k_{\perp}}^{2}+k_{\|}^{2}} e^{-2\left|k_{\|}\right| a}\left|j_{y}\right|^{2} d k_{\|}
$$

This expression highlights one of the fundamental challenges of low frequency wave heating, namely the fact that ingoing waves undergo a strong evanescence in the vacuum layer between the antenna and the plasma and are furthermore confronted on their path to the plasma bulk with brutal refractive index change, leading to a strong reflection at plasma vacuum interface. The $k_{\|}$spectrum of a given launcher is mostly determined by the geometry of the launcher. For a simple radiating strap of width $w$ it is given by $k_{\|} \sim \pi / w$. Therefore the radiated power will decay as $\exp (-2 \pi a / w)$. This provides a simple rule to estimate the evanescence of the field launched by the antenna in vacuum. If the launcher is made of an array of identical elements spaced by $L_{z}<w$ in the toroidal direction and excited with a phase difference $\Delta \Phi$, then the typical parallel wave vector must be taken as $k_{\|} a=\Delta \Phi / L_{z}$. Evanescence will then be stronger than in the previous case of an unphased structure. One could think that working with an antenna spectrum peaking at $k_{\|}=0$ (monopole phasing) could solve this problem. Actually realistic plasma surface spectra displays large contributions occurring 
for $k_{\|}<k_{0}$, but they are due to coaxial \& surface modes[14]. The coaxial modes correspond to TEM/z or $\mathrm{TE} / \mathrm{z}$ electromagnetic waves propagating between the tokamak wall and the plasma column, which plays the role of a lossy metal wall. These modes lead to power deposition in the SOL and wall region.

\section{G. Antenna coupling properties}

The structure of the antenna modelled in the present exercise is basically that of a strip-line, i.e. a conductor running above an infinite conducting plane. The field in such a strip line is known to have a TEM (transverse electro-magnetic) structure, like a coaxial transmission line. A TEM field has the property that the electric and magnetic field structure in the transmission line cross-section is the same as that respectively of the electrostatic and magnetostatic field it can sustain. Therefore, the antenna properties can be computed in the electrostatic and magnetostatic limits and used as in transmission line theory. We shall recast the results obtained above in terms of strip line characteristics. This formalism is often used in practice to represent properties of real ICRF antennas, which structure is indeed close to that of strip lines. The radiation impedance $Z_{A}$ of the antenna can be obtained by equating the power flow to the same expression for a transmission line:

$$
-\frac{1}{2} \int_{V} \vec{E} \cdot \overrightarrow{j_{A}} d V=\frac{1}{2}(R+i X) I^{2}
$$

where $I$ is the total current flowing on the antenna, $R$ and $X=-\omega L$ being respectively the specific input resistance and reactance of the line, and $L$ being the specific inductance. From the spectral form of Poynting's theorem (17) we obtain:

$$
\frac{1}{2}(R-i \omega L) I^{2}=-\frac{1}{4 \pi} \int_{-\infty}^{\infty} E_{y}\left(k_{\|}\right) j_{A}\left(k_{\|}\right) d k_{\|}
$$

The previous equality constitutes the definition of the two quantities $R$ and $L$ in the present antenna model. The plasma enters their definition only through the functions $g_{2}$ and $h_{2}(19 \mathrm{~b})$. No equivalent capacitance $C$ appears in Eq.(23) because we have dropped the TM part of the field. It can however easily be obtained by solving the TM vacuum field equations with the boundary condition $E_{y}=0$ at the plasma $x=0$. The three constants $R, L, C$ completely determine the properties of the transmission-line equivalent to the antenna $[11,12]$ and are of primary interest for the design of the transmission and matching system[2].

\section{H. Radiated field}

Using the additional boundary condition at the plasma-vacuum interface, i.e. continuity of $E_{y}$, the field in the plasma can also be computed and used to determine the properties of the radiated far field [15]. In Fig. 3, the far field Poynting flux distribution over constant phase surfaces is shown for a phased antenna array in an ITER-like plasma. As the Poynting flux is the RF power flux, this shows how phasing, by sending the power at different parallel wave numbers sends it in different spatial directions as well.

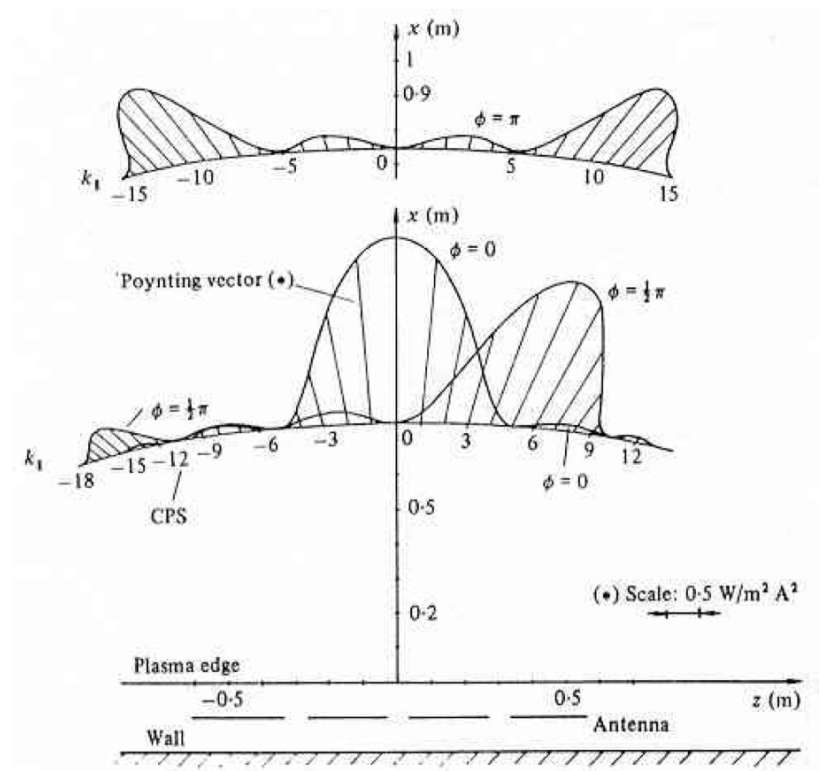

Figure 3: Distribution of the Poynting vector in the far-field region for three different phase differences $\Phi$ between successive straps of a 4-strap antenna array. From[15].

\section{State of the art}

The theory presented in the previous sections is as we said simple: it neglects feeders, poloidal antenna inhomogeneity and plasma density profile. These limitations were assessed in [12, 13] notably, and it appears that such a simple model can fairly reproduce most of the coupling properties of more realistic antenna models. Nevertheless the rigorous selfconsistent solution of the full boundary-values problem is a formidable task that can only be tackled with numerical codes. A review of the early development of sophisticated coupling codes can be found in the review[21] to which one must add reference to the RANT-3D code[20] describing coupling of recessed antennas to plasma. Both in ICANT[22] and in TOPICA[25] detailed current distributions are now computed over all antenna parts. In parallel to the development of these plasma coupling codes, sophisticated commercial codes have been used to compute and optimise the reactive properties of antennas in vacuum or in the presence of a dielectric[23, 26, 29]. In this process, it was shown that an adequately chosen dielectric can to some extent simulate coupling to a plasma[26]. Antenna models have been compared with success to experimental results in a number of cases, both in the ICRF[20, 25, 17, 18, 27] and Lower Hybrid range[19] and are used to predict the performance of ITER antennas[28, 29]. 


\section{POWER COUPLING FROM WAVES TO PAR- TICLES}

\section{A. Absorption mechanisms}

Once the wave has been launched in the plasma, one could think that it can be damped simply because the accelerated particles experience a drag due to collisions. This is in general not the case. In the bulk of a hot plasma, e.g. $T_{e} \simeq T_{i}=5 \mathrm{keV}, n=510^{19} \mathrm{~m}^{-} 3$ the collision frequency is

$$
\nu=2.910^{-12} n \ln \Lambda T^{-3 / 2}=20 \mathrm{kHz}
$$

In electromagnetic theory the ratio $\nu / \omega,(\omega=2 \pi f)$ is characteristic of the importance of dissipative effects due to collisions with respect to reactive, i.e. wave oscillation, energy. For small values of $\nu / \omega$, the motion is almost dissipation-less and huge fields and large perturbations in the particle motion are necessary if any significant amount of energy is to be damped in the plasma. Equation (24) implies that, at frequencies in the $\mathrm{MHz}$ range or higher, direct dissipation of the wave by collisions will be negligible. In order to magnify collisional absorption one has recourse to resonances. Under resonance conditions, a small excitation will create either a huge response in the particle's motion (wave-particle resonance) or large wave-field build-up (wave resonance). In Fig.1 the "absorption zone" is the region where such a resonance takes place (the shape is of course only symbolic).

\section{B. Wave resonances}

Assuming that $k_{\|}$is for the essential determined by the antenna system, and thus fixed, we shall characterise the resonance by $k_{\perp} \rightarrow \infty$. An example of wave resonance is that of the fast wave, Eq.(7), when $u=k_{0}^{2} \varepsilon_{\perp}-k_{\|}^{2}=0$. We notice that, as we proceed from the plasma edge to the plasma inside, e.g. along the major radius direction $(x), \varepsilon_{\perp}$ will vary because both the magnetic field and the plasma density vary. If the resonance is isolated from the cut-off, the wave is absorbed at resonance [8].Typically we have both a cut-off and a resonance in the plasma, with a regio between them where the wave is evanescent. If the distance between cut-off and resonance is not too large, it is possible for part of the wave energy to tunnel through the evanescent region. This back-to-back resonance and cut-off (assumed located at $x=0$ ) can be described by a simple wavenumber dependence of the form

$$
k_{\perp, F W}=k_{\infty}^{2}-\frac{\alpha}{x} \equiv K^{2}(x)
$$

with $\alpha$ a positive constant. Eq.(25) is often called Budden equation $[10,8]$. Except in the vicinity of $x=$ 0 , the wave is propagating with constant $k_{\perp} \simeq \pm k_{\infty}$. If the wave approaches the resonance from the left, it will slow-down more and more (its group velocity

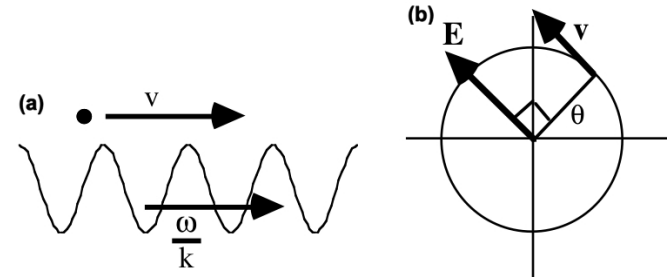

Figure 4: Wave-particle resonances: (a) Landau interaction; (b) cyclotron resonance

goes to zero) and come to rest at $x=0$. In this simple WKB picture, all wave fronts coming from the left pile-up at the resonance leading to a large increase in wave amplitude. In addition, as the wave field oscillates a large number of times before propagating any significant amount of distance toward the resonance, it is obvious that the effects of any damping mechanism will be considerably magnified in the vicinity of the resonance. A detailed integration of (25) in the complex plane shows that the power is constant for $x \neq 0$ and jumps abruptly at the crossing of the resonance. In the case of a cold or maxwellian plasma the jump correspond to a decrease in power, i.e. to wave energy absorption (by the plasma). This will be shown explicitly for the case of the ion-ion hybrid in a subsequent lecture [3]. Power is thus absorbed at the resonance notwithstanding the fact that no absorption mechanism was explicitly considered in the original equation (7).

\section{Wave-particle resonances - Landau damping}

Wave-particle resonances appear as resonant denominators in the integrand of the expression of the kinetic dielectric tensor[5]. Physically, they result from the fact that, in their reference frame, particles see a constant electric field and are therefore uniformly accelerated. Such a singular phenomenon can appear only in an approximate treatment of the problem, namely in the linearised approximation. For example let us consider, as in Fig.4-(a), a particle moving at the constant velocity $v$ in an electric field directed along $v$ and propagating at the phase velocity $\omega / k$. The equations of motion of this particle are

$$
m \frac{d v}{d t}=Z e E \cos (k v-\omega t) ; \frac{d x}{d t}=v
$$

When we linearise the problem $\left(v \simeq v_{0}+v_{1}\right)$ we arrive at the equation of motion

$$
\frac{d v_{1}}{d t}=\frac{Z e E}{m} \cos \left[\left(k v_{0}-\omega\right) t+k x_{0}\right]
$$

where we have used in the expression for the field the unperturbed particle motion $x=x_{0}+v_{0} t$. The perturbed velocity $v_{1}$ is oscillatory and there is no energy exchange between the particles and the wave as long as they have a different zero-order velocity $v \neq \omega / k$. But if they have the same velocity, the particle sees a 
constant electric field and is uniformly accelerated:

$$
v_{1}=\frac{Z e E}{m} \cos \left(k x_{0}\right) t
$$

An alternative way of looking at the same phenomenon is the Fourier transform approach, which here reduces to looking only at the periodic solutions of the problem:

$$
v_{1}=\frac{Z e E}{m} \frac{e^{i\left(k v_{0}-\omega\right) t}}{i\left(k v_{0}-\omega\right)}
$$

It can be seen that the singularity in the spectrum appearing at the resonance corresponds to the secularity $(\sim \mathrm{t})$ in the time-representation. The corresponding absorption is known as Landau damping and the condition $v=\omega / k$ is called the resonance condition. Whether the particle will be continuously accelerated or decelerated depends on the phase of the wave at the initial particles position $k x_{0}$. To see if globally the power will go from the wave to the particles or vice versa, we have to make an average of the energy increment over initial phases and over the initial particle distribution function[24], which leads to the classical Laudau's formula:

$$
\frac{d W}{d t} \approx-\frac{\pi \omega Z^{2} e^{2} E^{2}}{2 m k|k|} f^{\prime}\left(\frac{\omega}{k}\right)
$$

We see that damping rate is proportional to the slope of the unperturbed velocity distribution function at the phase velocity of the wave. Landau's formula (30) shows that power is transferred from the waves to the particles if there are more particles slower than the waves than faster ones. This is the case for a maxwellian. In the other case energy will go from the particles to the wave, which will be amplified, leading to instability (this process is sometimes called "inverse Landau damping"). A distribution function monotonous and decreasing constitutes therefore a dissipative medium in the absence of collisions.

D. Wave-particle resonances - Transit time magnetic pumping

Another frequently quoted non-collisional absorption mechanism is transit time magnetic pumping or TTMP. It is similar to Landau damping, except that it is due to a propagating magnetic rather than electricwave modulation $B_{1}=\delta B \cos (k x-\omega t)$ superposed on the static field $B_{0}$. This modulation gives rise to the perpendicular force $\mu \nabla B$ acting on the particles magnetic moment $\mu$, according to the equation for the motion along the magnetic field lines:

$$
m \frac{d v}{d t}=-\mu \nabla B
$$

which is similar to Eq.(26).

E. Wave-particle resonances - Cyclotron damping
Another type of wave-particle resonance is the cyclotron resonance (Fig.4-(b)). Assume that the particle is rotating at the cyclotron frequency $\theta=\omega_{c} t$ and that we apply a rotating electric field at the same frequency $\omega=\omega_{c}$ with a component along the particle velocity $v$ : the particles perpendicular energy will increase linearly with time. This mechanism will be discussed in details in [3].

This very sketchy analysis puts into light a weakness of the linearised approach. Indeed what will happen in reality is not that uniform acceleration will take place indefinitely but rather that the accelerated particle will escape the resonant condition and thence terminate the resonant process. Resonant absorption can only continue if, once this particle has left the interaction area, it is replaced by a fresh one that can continue the resonant interaction. In terms of distribution functions, this means that the wave will produce velocity diffusion such as to empty the interaction region from particles, which in practice means a flattening of the distribution function in the interaction zone. Once the distribution is flat the interaction has stopped. Collisions or other processes like stochasticity counteract this tendency by restoring the gradients. If the latter processes are strong, the distribution function can remain maxwellian, however, generally speaking, the distribution function of a particle population heated by a resonance process is not maxwellian.

\section{QUASILINEAR DIFFUSION AND TAILS}

In the previous sections, we have seen that the $\mathrm{RF}$ power could be absorbed by the plasma via waveparticle or wave resonances. In both cases, the wave equation tells us that the wave will be damped while travelling in the plasma but leaves open the question: where is this power going to ?

A wave resonance corresponds to infinity in physical variables. At resonance, not only the wave vector goes to infinity but field components as well, as is clear from the solution of the wave Eq.(7), which can be expressed analytically in terms of Bessel functions[8]. Such infinities are the sign that some smallness hypothesis is violated and that additional terms should have been retained in the wave equation. Retaining these terms changes the resonance into a mode conversion [9] whereby the initial low- $k_{\perp}$ wave is converted into high- $k_{\perp}$ branch. Ultimately, the latter can only be absorbed through collisional or non-collisional (i.e. wave-particle resonance) processes.

The existence of absorption through wave-particle resonance manifests itself by the presence of an antihermitian part of the magnetised hot-plasma dielectric tensor[5]. The classical expression of the dielectric tensor is obtained by assuming that the unperturbed distribution function is a maxwellian. We shall see 
that this is only an approximation and that the heating process only takes place with a sub-class of plasma particles and necessarily leads to some deformation of the distribution function of the heated population.

The corresponding theory is called quasilinear theory and we will here derive the equations for the simplest case, that of Landau damping in unmagnetised plasma. We start with Vlasovs equation in one dimension:

$$
\frac{\partial f}{\partial t}+v \frac{\partial f}{\partial z}+\frac{Z e E}{m} \frac{\partial f}{\partial v}=0
$$

where $E=E_{1}$ is the perturbed electric field (there is no equilibrium electric field). We decompose the distribution function into a slowly varying part $\left(f_{0}\right)$, both in time and space, and a perturbed part $\left(f_{1}\right)$, $f=f_{0}+f_{1}$, and insert this expression in Eq.(32). In order to isolate the slowly varying part of the distribution function, we average this equation over time (many wave periods) and space (many wavelengths). Denoting by $<$. $>$ this averaging operation, we obtain:

$$
\frac{\partial f_{0}}{\partial t}+v<\frac{\partial f_{0}}{\partial z}>+\frac{Z e}{m}<E \frac{\partial f_{1}}{\partial v}>=0
$$

where we have used the fact that $\left.\langle E\rangle=<f_{1}\right\rangle=0$. The second term in this equation is zero for uniform plasma and we are left with an equation that determines the evolution of the equilibrium distribution function under the action of the first-order perturbations:

$$
\frac{\partial f_{0}}{\partial t}=-\frac{Z e}{m}<E \frac{\partial f_{1}}{\partial v}>
$$

The second term in this equation is the quasilinear term. In order to write it down explicitly, we must solve the equation for the perturbation of the distribution function $f_{1}$ which we obtain by subtracting Eq.(34) from Eq.(32):

$$
\frac{\partial f_{1}}{\partial t}+v \frac{\partial f_{1}}{\partial z}+\frac{Z e E}{m} \frac{\partial f_{0}}{\partial v}=0
$$

Fourier-transforming in space and Laplacetransforming in time $(f \sim \exp [i(k z-\omega t)])$, we easily obtain the solution of this equation:

$$
f_{1}=-\frac{i Z e E}{m(\omega-k v)} \frac{\partial f_{0}}{\partial v}
$$

Inserting this expression into Eq.(34), we obtain, noting that the average of two oscillating quantities $u(t)$ and $v(t)$ is $\langle u(t) v(t)\rangle=(1 / 2) \Re\left(u v^{*}\right)$ :

$$
\frac{\partial f_{0}}{\partial t}=-\frac{Z^{2} e^{2}|E|^{2}}{2 m^{2}} \frac{\partial}{\partial v} \Im\left[\frac{1}{\omega-k v}\right] \frac{\partial f_{0}}{\partial v}
$$

This equation can be given a Fokker-Planck type form:

$$
\frac{\partial f_{0}}{\partial t}=\frac{\partial}{\partial v}\left(D \frac{\partial f_{0}}{\partial v}\right) ; D=-\frac{Z^{2} e^{2}|E|^{2}}{2 m^{2}} \Im\left[\frac{1}{\omega-k v}\right]
$$

where $D$ is the quasilinear diffusion coefficient. Note that the imaginary part appearing in the expression for $D$ is nothing else than $\delta(\omega-k v)$. This implies that only the particles which are in resonance will be pushed in velocity space by the heating process. If the wave is directional, i.e. if the $k_{\|}$spectrum is asymmetric, the deformation of the distribution function will also be asymmetric, leading to the generation of current by the waves, like in $\mathrm{LH}$ current drive.

\section{THERMALISATION}

If Eq.(38) is solved as it is, it can lead to a time-asymptotic stationary solution only if the energy transferred by the wave to the particle is zero. Therefore the stationary solution of Eq.(38) must exhibit a quasilinear plateau, i.e. a zones around the resonant velocity where $f_{0}$ is flat $\left(\partial f_{0} / \partial v=0\right)$. This ensures that the heating power vanishes.

In a situation where there is stationary power transfer to the plasma, the evolution equation for the particles distribution functions, Eq.(38) is thus lacking a loss term. This is the collision term, which we denote by $C\left(f_{0}\right)$. It includes collisions on all particle species, including the heated ones. It also implies that the distribution function of the heated species will tend to relax to a maxwellian. From the different contributions to $C\left(f_{0}\right)$, one can then compute the power transfer to the different plasma components and the resulting temperature increases. The complete equation for the evolution of $f_{0}$ should thus be written

$$
\frac{\partial f_{0}}{\partial t}=\frac{\partial}{\partial v}\left(D \frac{\partial f_{0}}{\partial v}\right)+C\left(f_{0}\right)
$$

This collisional thermalisation process is in close relation with the slowing down of fast NBI ions discussed in $[6]$.

\section{REFERENCES}

1. Y. Kazakov, "Heating the Plasma", These Proc.

2. P. Dumortier, "Antenna Design and Matching Issues", These Proc.

3. E. Lerche, "Ion cyclotron, lower hybrid and Alfvén wave heating", These Proc.

4. E. Westerhof, "Electron Cyclotron Resonance Heating", These Proc.

5. D. Van Eester \& E. Lerche, "Kinetic Theory of Plasma Waves", These Proc.

6. D. Van Eester, "Fast Particle Heating (NBI) \& Modelling particle heating and current drive in tokamaks", These Proc.

7. J.D. Jackson, "Classical Electrodynamics" 2nd Ed., J. Wiley \& Sons (1975) 
8. T.H. Stix, Waves in plasmas, AIP New York (1992).

9. D.G. Swanson, Plasmas Waves, American Institute of Physics (1989).

10. K.G. Budden, "The Propagation of Radio Waves", Cambridge U.P. (1985)

11. R.R. Weynants textitet al., "ICRH antenna design and coupling optimisation studies", in "Heating in toroidal plasmas", Commission of the European Communities, Vol. I (1980), 487

12. A. M. Messiaen et al., "Theoretical aspects of the coupling properties of ICRH antennae", in "Heating in toroidal plasmas", Commission of the European Communities, Vol. I (1982), 243

13. V.P. Bhatnagar et al., "A 3-D Analysis of the Coupling Characteristics of ICRH Antennae", Nucl. Fusion 22 (1982) 280

14. A. M. Messiaen et al., "Analysis of the plasma edge radiation by ICRH antenna", Proc. 4th International Symp. on Heating in Toroidal Plasmas, Vol. I (1984), 315

15. R. Koch et al., An asymptotic analysis of the radiation pattern of ion cyclotron resonance heating antennas, J. Plasma Phys. 32 (1984), 291

16. R. Koch, "A global solution of the ICRH problem based on the combined use of a planar coupling model and hot-plasma ray-tracing in tokamak geometry", Computer Phys. Comm., 40 (1986), 1

17. R. Koch et al., "A Comparison Between ICRF Theory and Experiment", Plasma Phys. Contr. Fus., 30 (1988), 1559

18. P. Descamps et al., "Global Modes Excitation in TEXTOR and Comparison with Theory", Plasma Phys. Contr. Fus., 33 (1991), 1109

19. C. Gormezano, "High power lower hybrid current drive on JET: results and prospects", Report JET-P(93)65 (1993)

20. M.D. Carter et al., "Three Dimensional Modelling of ICRF Launchers for Fusion Devices", Nucl. Fusion 36 (1996), 209,

21. R. Koch et al.,"Progress in RF Theory: a sketch of recent evolution in selected areas", J. Plasma Phys. 40 (1998), A141

22. S. Pecoul et al., "Numerical modeling of the coupling of an ICRH antenna with a plasma with selfconsistent antenna currents", Computer Phys. Comm., 146 (2002)166-187
23. D. Hartmann et al., "Measurements and calculations of electrical properties of ICRF antennas", in Radio Frequency Power in Plasmas, AIP Conf. Proc. 694 (2003), 106

24. R. Koch, "Wave-particle interactions in plasmas", Plasma Phys. Contr. Fus., 48(2006), B329

25. V. Lancellotti et al, "TOPICA: an accurate and efficient numerical tool for analysis and design of ICRH antennas", Nucl. Fusion 46 (2006), S476

26. P. Lamalle et al., "Recent developments in ICRF antenna modelling ", Nucl. Fusion, 46 (2006), 432-443

27. D. Milanesio et al., "Analysis of the Tore Supra ICRF antenna in TOPICA RF antenna code", Plasma Phys. Contr. Fus., 39 (2009), 405-419

28. D. Milanesio \& R. Maggiora, "ITER ICRF antenna analysis and optimisation using the TOPICA code", Nucl. Fusion 50 (2010), 025007

29. F. Louche et al., "3D electromagnetic optimization of the front face of the ITER ICRF antenna", Nucl. Fusion 51 (2011), 103002

30. A. Messiaen \& R. Weynants, "ICRH antenna coupling physics and optimum plasma edge density profile. Application to ITER", Plasma Phys. Contr. Fus. 53 (2010) 085020 